\title{
AVALIAÇÃo DE CULTIVARES DE BANANEIRA (Musa sp) NA REGIÃo DE SELVÍRIA-MS'
}

\author{
EDICLÉIA APARECIDA DA SILVA², APARECIDA CONCEIÇÃO BOLIANI ${ }^{3}$, LUIZ DE SOUZA CORRÊA ${ }^{3}$
}

\begin{abstract}
RESUMO - O presente trabalho teve como objetivo avaliar o ciclo e a produção de sete cultivares de bananeira na região de Selvíria, Estado do Mato Grosso do Sul. O delineamento experimental utilizado foi do tipo blocos ao acaso, com sete tratamentos (Nanica; Mysore; Marmelo; Ouro-da-Mata; Ourinho; São Tomé e Prata) e quatro repetições, num total de 28 parcelas. Os resultados obtidos permitiram as seguintes conclusões: A cultivar Mysore mostrou maior período do plantio ao florescimento, e as cultivares Nanica e São Tomé, menores. As cultivares Ouro-da-Mata e Ourinho apresentaram menor ciclo do plantio à colheita, enquanto as cultivares Mysore e Prata apresentaram ciclos maiores e iguais. As cultivares Marmelo e Nanica produziram cachos e pencas maiores, e as cultivares Ouro-da-Mata e Ourinho produziram cachos e pencas menores. A cultivar Mysore apresentou cacho com maior número de pencas, e a cultivar São Tomé, menor número. A cultivar Ourinho obteve maior número de frutos por penca, e a cultivar Marmelo, menor número. A cultivar Marmelo destacou-se com maior comprimento e diâmetro dos frutos da segunda penca. As melhores cultivares para esta região são Nanica e Marmelo, pois apresentaram bom desenvolvimento.
\end{abstract}

Termos para Indexação: ciclo de produção, cultivares de banana, características culturais.

\section{EVALUATION OF BANANA (Musa sp) CULTIVARS IN SELVÍRIA-MS REGION}

ABSTRACT - The objective of this study was to evaluate the development and the production of seven banana cultivars in the region of Selvíria, Mato Grosso do Sul state. The experiment was conducted in random blocks, under seven different treatments (Nanica; Mysore; Marmelo; Ouro-daMata; Ourinho; São Tomé e Prata) with four repetitions, in a total of 28 plots. The results led to the following conclusions: the period which goes from the planting to the flowering was longer for the Mysore cultivar and shorter for the Nanica and São Tomé cultivars. The development from the planting to the harvesting was shorter for the Ouro-da-Mata and Ourinho cultivars and equally longer for the Mysore and Prata cultivars. The Marmelo end Nanica cultivars produced bigger racemes and bunches while the Ouro-da-Mata and Ourinho cultivars produced smaller ones. The Mysore cultivar produced racemes with more bunches and the São Tomé cultivar produced racemes with fewer bunches. The longer and thicker fruits of the second bunch were found in the Marmelo cultivar. The best cultivars for this region are: Nanica and Marmelo as their development was better than the others.

Index terms: production cycle, banana cultivars, crop characteristics.

\section{INTRODUÇÃO}

A bananeira é originária do continente asiático, sendo atualmente distribuída em várias regiões tropicais, sendo que a área total e a produção no mundo é de 4.475 .831 ha; $69.832 .378 \mathrm{Mt}$ (FAO, 2003), respectivamente. Os principais países produtores são: Índia (620.000ha; 16.450.000Mt), Brasil (508.524ha; 6.369.450Mt), Filipinas (390.000ha; 5.264.470Mt), Burundi (300.000ha; $1.548 .897 \mathrm{Mt})$, Indonésia (284.000ha; 3.696.073Mt), China (263.967ha; 5.651.724Mt), Uganda (164.800ha; $988.80 \mathrm{Mt}$ ) e Tailândia (135.000 ha; $1.750 .000 \mathrm{Mt})$.

A banana é uma das frutas mais consumidas no mundo. Seu consumo diário é maior talvez que qualquer outra fruta, quer crua, quer cozida, assada ou frita. Sua produção comercial em plantações da América Central e da América do Sul reveste-se de uma grande importância, sendo hoje a terceira fruta no mundo em volume de produção, superada apenas pela uva e pela laranja. Além do alto valor nutritivo, a banana tem alto significado socioeconômico, pois mobiliza um grande contingente de mão-de-obra, permite retorno rápido ao produtor e é geradora de divisas para o País (GANGA, 2002).

No Brasil, a bananeira é cultivada em praticamente todos os Estados, destacando-se: São Paulo, Paraíba, Minas Gerais, Bahia, Santa Catarina, Amazonas, Ceará, Mato Grosso, Pernambuco e Espírito Santo (PEREZ, 2002), indo desde a faixa litorânea até os planaltos do interior, embora o seu plantio sofra restrições, em virtude de fatores climáticos, como temperatura e precipitação. São Paulo contribui com a maior produção por Estado (53.800 ha), com cerca de 1,1 milhão de toneladas por ano (AGRIANUAL 2005).

As cultivares mais difundidas são: Maçã, Prata, Pacovan, Prata-Anã, Mysore, Terra e D'Angola, pertencentes ao grupo genômico AAB, e Nanica, Nanicão e Grande Naine, do grupo AAA, utilizadas principalmente para exportação. As cultivares podem ser consumidas na forma in natura, doces, sorvetes, fritas ou cozidas (DANTAS \&

\section{SOARES FILHO, 1997).}

Os maiores problemas do cultivo da bananeira no Brasil são a falta de variedades comerciais produtivas, com porte adequado e resistência às principais pragas e doenças, além da inadequada condução do sistema solo-água-planta. A sigatoka-amarela é um agravante a mais, aliada à sigatoka-negra (recentemente introduzida) e mal-do-Panamá, que podem causar danos expressivos à bananicultura nacional, com perdas de até $100 \%$ na produtividade (TODA FRUTA, 2005).

Informações sobre algumas cultivares potenciais em diferentes regiões é de grande importância para o desenvolvimento da cultura, e, diante do exposto, o presente trabalho teve como objetivo avaliar sete cultivares potenciais de bananeira na região de Selvíria - MS.

\section{MATERIAL E MÉTODOS}

O trabalho foi conduzido na Fazenda de Ensino, Pesquisa e Extensão da Universidade Estadual Paulista "Júlio de Mesquita Filho" - UNESP, Câmpus de Ilha Solteira, localizada no município de Selvíria, Estado do Mato Grosso do Sul. O delineamento experimental utilizado foi do tipo blocos ao acaso, com sete tratamentos (Nanica; Mysore ; Marmelo; Ouro-da-Mata; Ourinho; São Tomé e Prata), 4 repetições e 4 plantas úteis por parcela.

De acordo com Embrapa (1999), o solo foi classificado como Latossolo Vermelho Distrófico típico argiloso, A moderado, hipodistrófico álico, caulinítico, férrico, compactado, muito profundo, moderadamente ácido (LVD).

O bananal foi implantado em julho de 2001, no espaçamento 3 x $2 \mathrm{~m}$, utilizando mudas do tipo rizoma não-brotado - inteiro, com peso de aproximadamente $2 \mathrm{~kg}$.

Os tratos culturais utilizados foram os convencionais, adotados para a cultura na região: a) plantio/cova : 300 g de calcário,

(Trabalho 128/2004). Recebido: 07/10/2004. Aceito para publicação: 20/02/2006.

2 Aluna do Mestrado do Programa de Pós-graduação em Agronomia da Universidade Estadual Paulista (Unesp) Câmpus de Ilha Solteira-SP, Passeio Prado, 317, Fone: (18)3742.3206, e-mail: edicleia.silva@bol.com.br.

${ }_{3}^{3}$ Docentes da Unesp, Departamento de Fitotecnia, Socioeconomia e Tecnologia de Alimento, Av. Brasil, 56, Ilha Solteira-SP, Fone: (18)3743.1243, e-mail: boliani@agr.feis.unesp.br; lcorrea@agr.feis.unesp.br. 
$500 \mathrm{~g}$ de superfosfato simples e $20 \mathrm{~L}$ de esterco de curral; b) adubação de formação: $100 \mathrm{~g}$ de sulfato de amônio, $170 \mathrm{~g}$ de superfosfato simples e $30 \mathrm{~g}$ de cloreto de potássio, sendo que a distribuição foi feita a lanço, ao redor da cova, trinta e sessenta dias após o plantio; c) o sistema de irrigação adotado foi do tipo gotejamento; d) o controle de plantas daninhas foi realizado através de capinas manuais e do herbicida Gramoxone (Paraquat), e e) o controle fitossanitário foi realizado através de aplicações com Furadan $50 \mathrm{G}$ (Carbofuran) (30 g por cova: em sulco ao redor das plantas), visando principalmente ao controle da Broca-daBananeira (Cosmopolites sordidus) e Nematóides (Radopholus similis), e para a doença Mal-de-Sigatoka (Mycosphaerella musicola Leach) foi utilizado Óleo Mineral, Benlate ( Benomyl: na dosagem de $60 \mathrm{~g} / 100 \mathrm{~L}$ de água) e Cercobin (Tiofanato Metílico: na dosagem de 70g/100L de água).

As características avaliadas foram: período do plantio ao florescimento; abertura das primeiras brácteas; emissão do cacho-corte do coração; corte do coração à colheita; período do plantio à colheita e características dos cachos: peso do cacho; peso da segunda penca; número de pencas por cacho; número de frutos por penca; comprimento e diâmetro médio dos frutos da segunda penca.

Os dados foram tabulados através do programa Estat, sendo analisados quadrados médios das análises de variância e níveis de significância referentes às características avaliadas através do teste de Tukey $(\mathrm{P}<0,05)$.

\section{RESULTADOS E DISCUSSÃO}

Através da Tabela 1, verifica-se que houve diferença altamente significativa a $1 \%$ para as características período do plantio ao florescimento, emissão do cacho - corte do coração, corte do coração - colheita, período do plantio à colheita e diferença significativa a $5 \%$ apenas para abertura das primeiras brácteas. A Tabela 2 apresenta os resultados relativos às características peso do cacho, peso da segunda penca, número de pencas por cacho, número de frutos por penca, comprimento e diâmetro médio dos frutos da segunda penca, os quais foram altamente significativos $(\mathrm{P}<0,01)$. Observa-se que, para a característica período do plantio ao

TABELA 1 - Quadrados médios de tratamentos (cultivares) referentes às caraterísticas: período do plantio ao florescimento $(\mathrm{P}-$ $\mathrm{F})$; abertura das primeiras brácteas ( $\mathrm{APB})$; emissão do cacho - corte do coração (EC-CC) ; corte do coração à colheita (CC-C), e período do plantio à colheita ( $\mathrm{P}-\mathrm{C})$. Selvíria-MS, 2002.

\begin{tabular}{cccccc}
\hline & \multicolumn{5}{c}{ Características } \\
\cline { 2 - 6 } $\begin{array}{c}\text { Causas de } \\
\text { variação }\end{array}$ & P-F & A P B & EC-CC & CC-C & PP-C \\
\hline Cultivares & $4,98^{* *}$ & $2,47^{*}$ & $5,76^{* *}$ & $472,09^{* *}$ & $5,00^{* *}$ \\
Resíduo & ------ & ------- & ------ & ------ & ------ \\
\hline CV(\%) & 7,76 & 35,00 & 14,04 & 2,35 & 7,78 \\
\hline
\end{tabular}

*Significativo ao nível de $5 \%$ de probabilidade $(\mathrm{P}<0,05)$; * Significativo ao nível de $1 \%$ de probabilidade $(\mathrm{P}<0,01)$. florescimento, a cultivar Nanica apresentou menor período (206 dias), diferindo das cultivares Prata (250,75 dias) e Mysore (263 dias). Estes valores são semelhantes (270 dias) aos encontrados por Perez (2002).

Observa-se que, para a característica abertura das primeiras brácteas, a cultivar Mysore apresentou o menor valor (10 dias), diferindo apenas da cultivar Nanica (21,75 dias), que apresentou período maior e semelhante (24 dias) ao encontrado por Ganga (2002). O período entre emissão do cacho - corte do coração das cultivares Mysore (11,75 dias), Prata (11 dias), Ourinho (12 dias), São Tomé (13 dias) e Marmelo (11 dias) apresentaram valores significativamente superiores. As cultivares Ouro-da-Mata e Ourinho apresentaram menores períodos para a característica corte do coração à colheita (54 e 57 dias, respectivamente).

Verifica-se, na Tabela 3, que as cultivares Ouro-da-Mata e Ourinho foram as que apresentaram menores ciclos (288 e 291 dias, respectivamente), enquanto as cultivares Mysore e Prata apresentaram ciclos maiores e iguais (396 dias), diferindo do resultado obtido (418 dias) por Dantas \& Soares Filho (1997).

Verifica-se, pela Tabela 4, que as cultivares Marmelo e Nanica produziram cachos maiores, com valores médios de 23,166 kg e 19,462 $\mathrm{kg}$, respectivamente, sendo estes maiores do que aqueles obtidos por Silva \& Alves (1999) (21,2 kg e 12,63 kg), respectivamente. Para a característica peso da segunda penca, as cultivares Marmelo e Nanica produziram pencas maiores $(3,650 \mathrm{~kg}$ e $2,950 \mathrm{~kg}$ respectivamente), valores estes próximos aos encontrados (4 kg e 3 $\mathrm{kg}$ respectivamente) por Saes \& Moreira (1998). A cultivar Mysore produziu maior número de pencas por cacho $(13,75)$ quando comparada às cultivares Nanica $(8,50)$ e Prata $(8,00)$. A cultivar Ourinho produziu maior número de frutos por penca $(18,75)$. Para as características comprimento e diâmetro médio dos frutos da segunda penca, a cultivar Marmelo apresentou maior comprimento e diâmetro médio $(18,25 \mathrm{~cm} ; 4,82 \mathrm{~cm}$, respectivamente), sendo significativamente superior às demais cultivares. Observa-se que pode ocorrer diferença entre cultivares e entre regiões devido ao tipo e idade da muda, bem como pelas condições ambientais, como altitude, luminosidade, temperatura e umidade (SIMÃO, 1998).

TABELA 2 - Quadrados médios de tratamentos (cultivares) referentes às caraterísticas: peso do cacho (PC); peso da segunda penca (PSP); número de pencas por cacho (NPC); número de frutos por penca (NFP); comprimento de frutos da segunda penca (CFSP), e diâmetro médio dos frutos da segunda penca (DMFSP). Selvíria-MS, 2002.

\begin{tabular}{ccccccc}
\hline & \multicolumn{7}{c}{ Características } \\
\cline { 2 - 7 } $\begin{array}{c}\text { Causas de } \\
\text { variação }\end{array}$ & PC & PSP & NPC & NFP & CFSP & DMFSP \\
\hline Cultivares & $31,59 * *$ & $26,98^{* *}$ & $36,24^{* *}$ & $13,80^{* *}$ & $39,96^{* *}$ & $20,43^{* *}$ \\
Resíduos & ------ & ----- & ----- & ----- & ----- & ----- \\
\hline CV $(\%)$ & 16,61 & 21,50 & 9,26 & 8,24 & 10,35 & 7,70 \\
\hline
\end{tabular}

*Significativo ao nível de $5 \%$ de probabilidade; ** Significativo ao nível de $1 \%$ de probabilidade.

TABELA 3 - Valores médios em dias para as características: período do plantio ao florescimento (P-F); abertura das primeiras brácteas (APB); emissão do cacho - corte do coração (EC-CC); corte do coração à colheita (CC-C), e período do plantio à colheita (P-C). Selvíria-MS, 2002.

\begin{tabular}{|c|c|c|c|c|c|}
\hline \multirow[t]{2}{*}{ Cultivares } & P-F & $\mathrm{APB}$ & $\mathrm{EC}-\mathrm{CC}$ & $\mathrm{CC}-\mathrm{C}$ & $\mathrm{P}-\mathrm{C}$ \\
\hline & \multicolumn{5}{|c|}{ Valores médios em dias } \\
\hline 1-Mysore & $263,00 \mathrm{~A}$ & $10,00 \quad \mathrm{~B}$ & $11,75 \mathrm{~A}$ & $81,00 \mathrm{C}$ & $396 \mathrm{~A}$ \\
\hline 2-Prata & $250,75 \mathrm{~A}$ & $12,00 \mathrm{AB}$ & $11,00 \mathrm{~A}$ & $119,00 \mathrm{~A}$ & $396 \mathrm{~A}$ \\
\hline 3-São Tomé & $207,25 \quad \mathrm{~B}$ & $12,50 \mathrm{AB}$ & $13,00 \mathrm{~A}$ & $75,50 \mathrm{D}$ & $356 \mathrm{AB}$ \\
\hline 4-Nanica & $206,00 \quad B$ & $21,75 \mathrm{~A}$ & $9,75 \mathrm{AB}$ & $83,00 \mathrm{C}$ & $333 \mathrm{AB}$ \\
\hline 5-Ouro da Mata & $244,50 \mathrm{AB}$ & $16,75 \mathrm{AB}$ & $7,00 \quad \mathrm{~B}$ & $54,00 \mathrm{E}$ & $288 \quad \mathrm{~B}$ \\
\hline 6-Ourinho & $232,25 \mathrm{AB}$ & $11,50 \mathrm{AB}$ & $12,00 \mathrm{~A}$ & $57,00 \mathrm{E}$ & 291 \\
\hline 7-Marmelo & $234,75 \mathrm{AB}$ & $14,25 \mathrm{AB}$ & $11,00 \mathrm{~A}$ & $93,00 \mathrm{~B}$ & $353 \mathrm{~A}$ \\
\hline
\end{tabular}

*Médias seguidas por letras distintas, nas colunas, diferem entre si, a 5\% de probabilidade, pelo teste de Tukey. 
TABELA 4 - Valores Médios para as características: peso do cacho (PC); peso da segunda penca (PSP); número de pencas por cacho (NPC); número de frutos por penca (NFP); comprimento de frutos da segunda penca (CFSP), e diâmetro médio dos frutos da segunda penca (DMFSP). Selvíria-MS, 2002.

\begin{tabular}{|c|c|c|c|c|c|c|}
\hline \multirow[b]{2}{*}{ Cultivares } & \multicolumn{6}{|c|}{ Característica } \\
\hline & $\mathrm{PC}(\mathrm{Kg})$ & PSP (Kg) & $\mathrm{NPC}$ & NFP & CFSP $(\mathrm{cm})$ & DMFSP $(\mathrm{cm})$ \\
\hline 1-Mysore & $14,166 \mathrm{~B}$ & $1,616 \mathrm{~B}$ & $13,75 \mathrm{~A}$ & $17,75 \mathrm{AB}$ & $7,75 \mathrm{E}$ & $3,27 \mathrm{CDE}$ \\
\hline 2-Prata & $11,383 \mathrm{BC}$ & $1,403 \mathrm{~B}$ & $8,00 \mathrm{~B}$ & $12,75 \mathrm{AB}$ & $13,25 \mathrm{BC}$ & $3,75 \mathrm{BC}$ \\
\hline 3-São Tomé & $10,183 \mathrm{BCD}$ & $1,616 \mathrm{~B}$ & $7,75 \mathrm{~B}$ & $14,25 \mathrm{C}$ & $11,25 \mathrm{CD}$ & $3,57 \mathrm{DE}$ \\
\hline 4-Nanica & $19,462 \mathrm{~A}$ & $2,950 \mathrm{~A}$ & $8,50 \mathrm{~B}$ & $15,00 \mathrm{BC}$ & $14,00 \mathrm{~B}$ & $3,87 \mathrm{BC}$ \\
\hline 5-Ouro-da-Mata & $5,616 \mathrm{~B}$ & $0,878 \mathrm{~B}$ & $7,00 \mathrm{~B}$ & $17,25 \mathrm{AB}$ & $8,25 \mathrm{E}$ & $2,72 \mathrm{E}$ \\
\hline 6-Ourinho & $7,212 \mathrm{CD}$ & $1,090 \mathrm{~B}$ & $6,75 \mathrm{~B}$ & $18,75 \mathrm{~A}$ & $8,00 \mathrm{E}$ & $3,12 \mathrm{E}$ \\
\hline 7-Marmelo & $23,166 \mathrm{~A}$ & $3,650 \mathrm{~A}$ & $7,25 \mathrm{~B}$ & $12,25 \mathrm{C}$ & $18,25 \mathrm{~A}$ & $4,82 \mathrm{~A}$ \\
\hline
\end{tabular}

* Médias seguidas por letras distintas, nas colunas, diferem entre si, a 5\% de probabilidade, pelo teste de Tukey.

\section{CONCLUSÕES}

Nas condições em que foi conduzido o trabalho, os resultados obtidos permitiram concluir que:

1. As cultivares Mysore, Nanica, Marmelo e Prata destacaramse por reunirem melhores características produtivas e atributos de qualidade.

2. O cultivo de bananas na região de Selvíria-MS, permite a obtenção de produtividades razoáveis e frutos de boa qualidade.

\section{REFERÊNCIAS}

AGRIANUAL 2005: anuário da agricultura brasileira. São Paulo: FNP Consultoria \& Comércio, 2005. p.220-229.

DANTAS, J. L. L.; SOARES FILHO, W. S.S. Classificação botânica, origem e evolução. In:__. Banana para exportação: aspectos técnicos da produção. 2.ed. Brasília: Embrapa/SPI, 1997. p. 9-12. (Série Publicações Técnicas FRUPEX, 18).

EMBRAPA. Sistema brasileiro de classificação de solo. Rio de Janeiro: Embrapa-CNPSO, 1999.p.1-42.
FOOD AGRICULTURE ORGANIZATION - FAO. Países produtores de banana. Disponível em: < http://apps.fao.org/lim500/nph wrap.pl >. Acesso em : 01 ago. 2003.

GANGA, R.M.D. Resultados parciais sobre o comportamento de seis cultivares de banana (Musa spp) em Jaboticabal. In: CONGRESSO BRASILEIRO DE FRUTICULTURA, 17., 2002, Belém. Anais... Belém Embrapa/DDT, 2002. CD- ROM

PEREZ, L.H. Distribuição geográfica da bananicultura no Estado de São Paulo, 1983-2001. Informações Econômicas, São Paulo, v.32, n.4, p.41, 2002.

SIMÃO, S. Tratado de fruticultura. Piracicaba: FEALQ, 1998. $760 \mathrm{p}$.

SILVA, O . S.; ALVES, E. J. Melhoramento genético e novas cultivares de bananeira. Banana: produção, colheita e pós-colheita. Informe Agropecuário, Belo Horizonte, v.20, n. 196.96 p, 1999.

SAES, L.; MOREIRA, R. Banana: principais cultivares brasileiras. Toda Fruta, São Paulo, v.3, n.5, p. 18-21, 1998.

TODA FRUTA, Características e doenças da banana. Disponível em: $<$ http://www.todafruta.com.br $>$. Acesso em: 18 jun. 2005. 